\title{
Platelet testing to guide aspirin dose adjustment in pediatric patients after cardiac surgery
}

\author{
Sirisha Emani, $\mathrm{PhD},{ }^{\mathrm{a}}$ David Zurakowski, $\mathrm{PhD},{ }^{\mathrm{b}}$ Michelle Mulone, BS, ${ }^{\mathrm{c}}$ James A. DiNardo, MD, \\ Cameron C. Trenor III, MD, MMSc, ${ }^{\mathrm{d}}$ and Sitaram M. Emani, MD $^{\mathrm{a}}$
}

\section{ABSTRACT}

Objectives: Thrombosis is associated with increased morbidity and mortality in pediatric patients undergoing cardiac surgery. Although aspirin commonly is used for thromboprophylaxis, the utility of laboratory-based tests that assess aspirin efficacy have not been evaluated. We sought to determine the relationship between platelet aggregation testing and aspirin dose adjustment on thrombosis rates in this population.

Methods: Pediatric patients undergoing cardiac surgery who received aspirin and underwent platelet testing were studied retrospectively. Patients were excluded if they were treated with multiple agents or experienced thrombosis before the initiation of aspirin. Thrombosis events within 30 days after initiation of aspirin were recorded. Associations between aspirin responsiveness and thrombosis rate and aspirin dose adjustment and thrombosis rate were assessed with the use of multivariable logistic regression analysis.

Results: Suboptimal platelet response to aspirin was detected in 64 of 430 patients $(15 \%)$ and thrombosis was detected in 11 patients $(2.6 \%)$. Lack of aspirin responsiveness on initial testing was a significant risk factor for thrombosis $(P<.001)$ independent of age, weight, diagnosis, and initial aspirin dose. Dose escalation based on aspirin testing was performed in 40 of 64 patients, and significantly lower rate of thrombosis was observed in patients who underwent dose escalation compared with those without dose escalation (0/40 vs 9/24, $P<.001)$. By multivariable analysis, the only significant independent risk factor for thrombosis was failure to increase aspirin dose after initial unresponsiveness $(P<.001)$.

Conclusions: Current practice of weight-based aspirin dosing may lead to subtherapeutic platelet inhibition in some children. Aspirin unresponsiveness is associated with increased risk of thrombosis after specific pediatric cardiac surgical procedures. Aspirin dose increase in unresponsive patients is associated with reduced risk of thrombosis. (J Thorac Cardiovasc Surg 2017;154:1723-30)

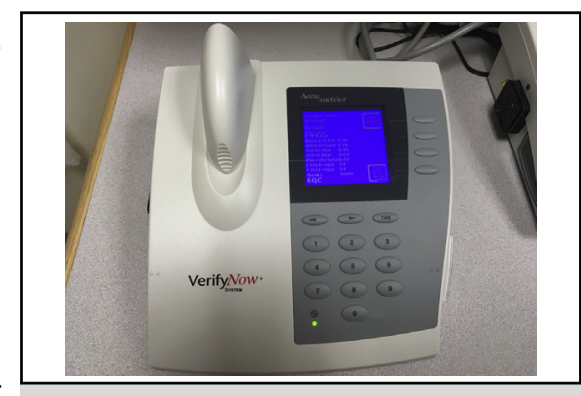

The Verify Now device allows automated testing of aspirin responsiveness in pediatric patients.

\section{Central Message}

Aspirin dose adjustment based on laboratory testing is associated with reduced risk of thrombosis in pediatric patients after cardiac surgery.

\section{Perspective}

Aspirin therapy is used frequently in pediatric patients after cardiac surgery. This study assesses the relationship between laboratory testing and clinical outcome of thrombosis in pediatric patients on aspirin therapy. A significantly greater rate of thrombosis was observed in patients with inadequate platelet inhibition, whereas dose escalation was associated with reduced thrombosis rate.

See Editorial Commentary page 1731.

See Editorial page 1714.
Rates of thrombosis in pediatric patients undergoing congenital cardiac surgery range from $5 \%$ to $20 \%$, and risk factors for thrombosis include neonatal surgery, single-ventricle physiology, and procedures that require

\footnotetext{
From the Departments of ${ }^{\mathrm{a}}$ Cardiovascular Surgery and ${ }^{\mathrm{b}}$ Anesthesia, ${ }^{\mathrm{c}}$ Cardiovascular Program Coagulation Laboratory, and ${ }^{\mathrm{d}}$ Division of Hematology/Oncology, Boston Children's Hospital, Harvard Medical School, Boston, Mass.

This study was supported by funding from the Goldwin Foundation, Lincoln, Neb. Received for publication Aug 31, 2016; revisions received March 21, 2017; accepted for publication June 13, 2017; available ahead of print July 13, 2017.

Address for reprints: Sitaram M. Emani, MD, 300 Longwood Ave, Bader 273,

Boston, MA 02115 (E-mail: sitaram.emani@cardio.chboston.org). $0022-5223 / \$ 36.00$

Copyright (c) 2017 by The American Association for Thoracic Surgery http://dx.doi.org/10.1016/j.jtcvs.2017.06.031
}

insertion of prosthetic material into the circulation (shunts, baffles, or valves) or significant reconstruction of coronary arteries. $^{1}$ Patients considered to be at high risk for thrombosis after these procedures receive prophylaxis with aspirin $(3-10 \mathrm{mg} / \mathrm{kg} / \mathrm{d})$ for several months to years postoperatively, and aspirin therapy is associated with reduced risk of thrombosis. ${ }^{2}$ The efficacy of aspirin to






$$
\begin{aligned}
& \text { Abbreviations and Acronyms } \\
& \text { AA = arachidonic acid } \\
& \text { ARU = aspirin reaction units } \\
& \text { AUC }=\text { Area under the curve } \\
& \text { CI = confidence interval } \\
& \text { CLIA = Clinical Laboratory Improvement } \\
& \text { Amendments } \\
& \text { IQR = interquartile range } \\
& \text { TEG-PM }=\text { thromboelastography with platelet } \\
& \text { mapping } \\
& \mathrm{UFH}=\text { unfractionated heparin }
\end{aligned}
$$

inhibit platelets can be assessed by assays that examine platelet aggregation in response to exogenous arachidonic acid (AA). Greater than $50 \%$ inhibition of AA-mediated platelet aggregation generally is considered to be indicative of laboratory testing-based aspirin responsiveness. ${ }^{3}$

Previous studies have highlighted significant interpatient variability in platelet response to weight-based aspirin dosing, with rates of aspirin unresponsiveness ranging from $10 \%$ to $26 \%{ }^{4,5}$ Heterogeneity in aspirin response may reflect the inherent variability in platelet function across individuals. ${ }^{6,7}$ Consistent with these findings, a recent prospective study in pediatric patients after high-risk cardiac surgery demonstrated a correlation of laboratory-based platelet inhibition with clinical outcomes of postoperative thrombosis. ${ }^{8}$ However, the impact of testing-based aspirin dose titration on the incidence of postoperative thrombosis has not been studied.

The purpose of this study was to determine the association between platelet testing and thrombosis rates in pediatric patients at high risk for thrombosis after cardiac surgery. Furthermore, we sought to determine the differences in thrombosis rates between patients in whom platelet testing was used to escalate aspirin dose compared with patients in whom aspirin dosing was weight-based.

\section{METHODS}

\section{Patients and Study Design}

On the basis of previous observational studies, a clinical protocol was developed at Boston Children's Hospital to provide aspirin responsiveness testing in patients undergoing high-risk pediatric cardiac surgery beginning in 2013. Pediatric patients (age $<18$ years) with congenital heart disease undergoing cardiac surgery at Boston Children's Hospital between January 1, 2013, and February 15, 2016, were identified. Patients who were considered to be at high risk for thrombosis, received single-agent antiplatelet therapy with aspirin, and underwent aspirin responsiveness testing were analyzed retrospectively. Patients were included in the study if they were considered to be at high risk for postoperative thrombosis due to one or more of the following criteria: (1) age < 30 days at surgery; (2) single-ventricle physiology undergoing palliative surgery; (3) congenital heart disease requiring complex valve repair/replacement, intracardiac baffling, or ventricular assist device; or (4) surgery for coronary artery reconstruction. Patients were excluded if they underwent multimodal therapy with concomitant antiplatelet or anticoagulant medications

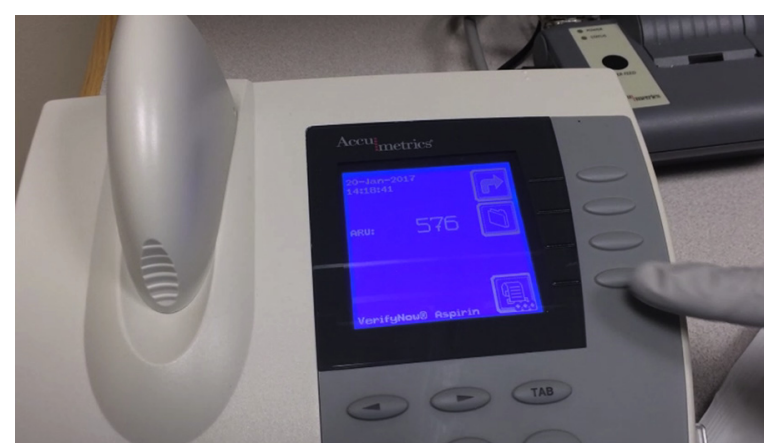

VIDEO 1. Testing of aspirin responsiveness on the VerifyNow device. The video describes the basic operational testing procedure for assessing platelet function on blood sample using the VerifyNow system. Video available at: http://www.jtcvsonline.org/article/S0022-5223(17)31347-8/fulltext.

(clopidogrel, low-molecular-weight heparin, or warfarin) or if they had a documented history of thrombosis before surgery. Patients were further excluded if thrombosis was documented after surgery but before aspirin administration or if aspirin was discontinued during their hospital course because of bleeding or other side effects. This study was approved by the Boston Children's Hospital Institutional Review Board.

\section{Anticoagulation Strategy}

After cessation of bleeding after cardiac surgical procedure, patients received unfractionated heparin (UFH) for a period of 2 to 5 days until removal of central venous catheters. Although most patients received low-dose UFH (10 U/kg/h), patients undergoing aortopulmonary shunting for single-ventricle palliation or ventricular assist device received therapeutic UFH. After the initiation of parenteral feeding, aspirin (3-10 $\mathrm{mg} / \mathrm{kg} / \mathrm{d}$ ) was administered either orally or via nasogastric tube. Aspirin was not initiated until resolution of thrombocytopenia (platelet count $>100)$. Doses typically were administered as fractions or multiples of $81 \mathrm{mg}(20.25,40.5,81$, or $162 \mathrm{mg})$ or $325-\mathrm{mg}$ tablets.

\section{VerifyNow Aspirin Responsiveness Testing}

Laboratory-based testing for aspirin responsiveness was performed after at least 2 doses of aspirin had been administered. Testing was performed in a Clinical Laboratory Improvement Amendments (CLIA)-certified laboratory (Cardiovascular Program Coagulation Laboratory) within Boston Children's Hospital. Aspirin responsiveness was measured with the VerifyNow system (Accumetrics Corp, San Diego, Calif) for quantitative measurement of platelet aggregation in response to exogenous AA as pervious described (Video 1). ${ }^{9}$ Values $<550$ aspirin reaction units (ARU) indicate impaired platelet aggregation in response to AA, suggesting therapeutic platelet inhibition; conversely, ARU $\geq 550$ indicates subtherapeutic platelet inhibition. ${ }^{9}$

\section{Thromboelastography With Platelet Mapping}

A subset of patients early in the study period underwent additional platelet testing with thromboelastography with platelet mapping (TEG-PM; Haemonetics Corporation, Braintree, Mass) to validate results of VerifyNow testing when it was first introduced into clinical practice. Blood samples for the TEG-PM were obtained and tested as per the manufacturer's protocol.

\section{Aspirin Dose Adjustment}

Therapeutic decisions regarding dose adjustment were not prescribed by clinical protocol, although clinicians were provided a recommended algorithm for incremental dose adjustment based on VerifyNow as follows: if $\mathrm{ARU} \geq 550$ at daily doses of $20.25 \mathrm{mg}, 40.5 \mathrm{mg}$, or $81 \mathrm{mg}$, then dose was 


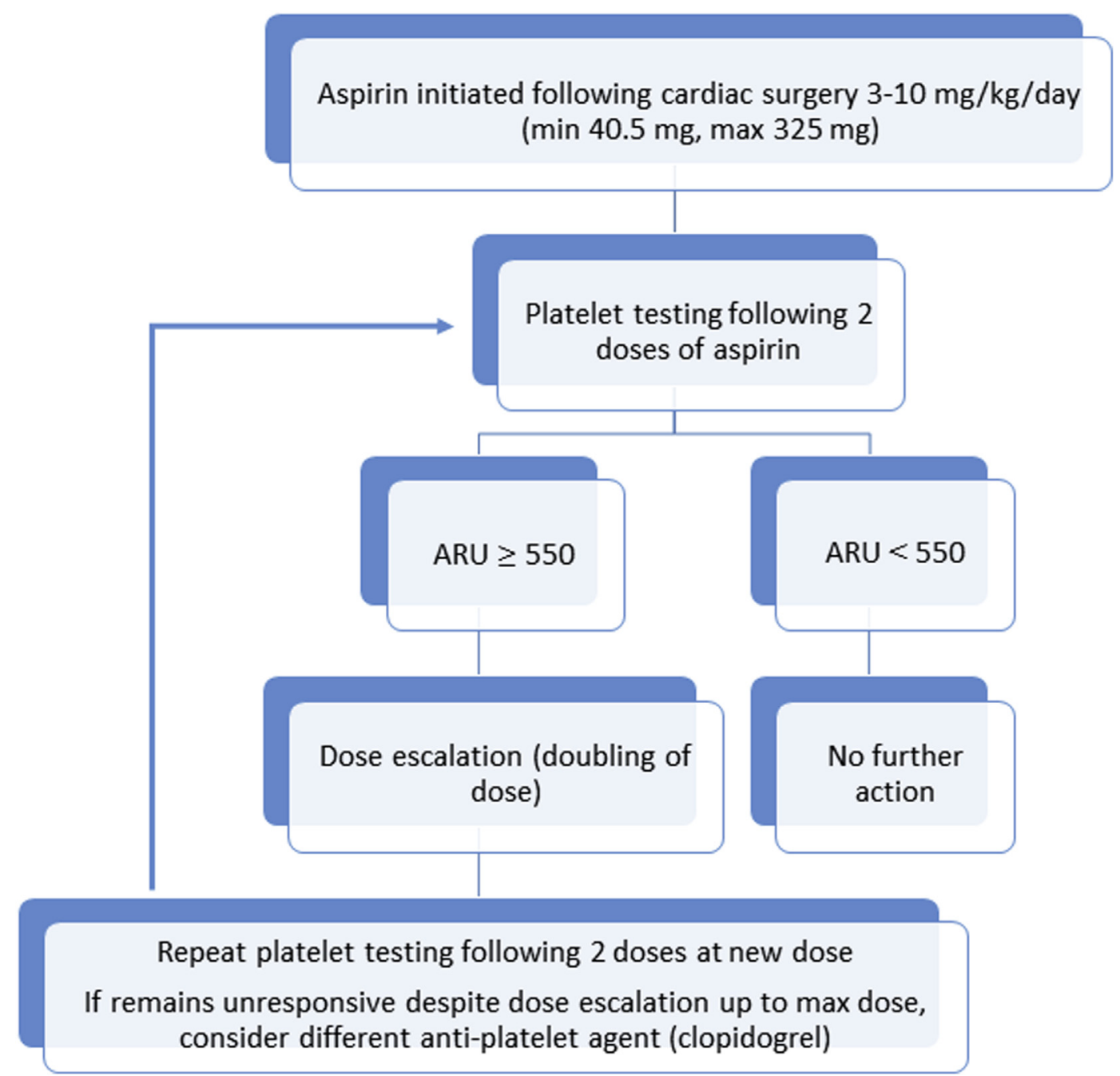

FIGURE 1. Schematic algorithm outlining recommended management of aspirin therapy in pediatric patients after high-risk cardiac surgery. $A R U$, Aspirin reaction units.

doubled. For patients with ARU $\geq 550$ at $162 \mathrm{mg} / \mathrm{d}$, dose was increased to $325 \mathrm{mg} / \mathrm{d}$. The recommended algorithm for aspirin dose adjustment is depicted in Figure 1. Repeat VerifyNow testing was recommended to confirm platelet inhibition, and if ARU $\geq 550$, repeat dose escalation up to a maximum dose of $10 \mathrm{mg} / \mathrm{kg} / \mathrm{d}$ or $325 \mathrm{mg} / \mathrm{d}$ was recommended. Aspirin resistance was defined as persistence of ARU $\geq 550$ despite a maximum dose of aspirin.

\section{Retrospective Data Collection}

All patients who had undergone testing with VerifyNow were screened according to inclusion and exclusion criteria after a review of electronic medical record. Demographic data, diagnosis, surgical procedure, and dose(s) of aspirin were recorded. Thrombosis events within 30 days of initiation of aspirin were determined by review of clinician inpatient and outpatient notes, discharge summaries, and imaging studies. As part of a standard clinical protocol, all patients obtained an echocardiogram within 2 weeks after surgery or before discharge, and were seen in clinic within 2 weeks after discharge. Symptomatic clinical thrombosis events (stroke, shunt thrombosis, limb ischemia) and asymptomatic evidence of thrombosis detected by imaging studies (echocardiography or cardiac catheterization) were recorded, and the interval to the event was documented.

\section{Statistical Analysis}

Univariate comparisons between patients with and without thrombosis (cases and controls) were performed with the nonparametric Mann-Whitney $U$ test for continuous variables including age, body weight, initial aspirin dose, and ARU reading and summarized by the median and interquartile range (IQR). Categorical data were compared with the Fisher exact test for binary proportions. Confidence intervals (CIs) for simple binary proportions were determined with the Wilson method with continuity correction. ${ }^{10}$ Multivariable analysis was conducted by logistic regression with the likelihood ratio test to assess significance of possible risk factors for thrombosis; this was done for the entire cohort of 430 patients as well as the subset of 64 patients who initially were unresponsive to aspirin to evaluate the relationship between dose increase and risk of thrombosis. ${ }^{11}$ Receiver operating characteristic curve analysis was applied to ascertain the correlation between initial ARU reading and thrombosis. Area under the curve (AUC) with the $95 \%$ CI was used to measure accuracy of the relationship between sensitivity (correctly classifying patients with thrombosis) and specificity (correctly classifying patients without thrombosis) as well as to identify an optimal cut-off value of ARU for balancing these 2 important characteristics, with an AUC of 0.800 or higher considered signficant. ${ }^{12-14}$ Analysis of the data was performed with IBM SPSS Statistics for Windows (Version 23.0, IBM 
Corp, Armonk, NY). Two-tailed values of $P<.05$ were considered statistically significant for all tests.

\section{RESULTS \\ Patient Characteristics}

Within the study period, 456 patients met inclusion criteria; the rate of thrombosis within 30 days postsurgery in this cohort of pediatric patients undergoing cardiac surgery was $7.5 \%$ (34/456). Only 430 were considered in the subsequent analyses because 23 patients experienced thrombosis before initiation of aspirin and 3 required discontinuation of aspirin due to bleeding or side effects (gastritis or necrotizing enterocolitis). The general characteristics of the cohort of 430 patients used for subsequent analysis are shown in Table 1 . Thrombosis within 30 days of surgery was observed in 11 of $430(2.6 \%)$ patients. Location of thrombosis was the femoral artery $(n=2)$, Fontan pathway/fenestration $(n=1)$, embolic stroke $(n=2)$, left ventricle $(n=1)$, right atrium/superior vena cava $(n=2)$, aortopulmonary shunt $(\mathrm{n}=2)$, and coronary artery anastomosis $(\mathrm{n}=1)$. Within the cohort of 430 patients, aspirin unresponsiveness on initial postoperative testing was detected in 64 patients $(15 \%)$ (Table 1).

\section{Relationship Between Aspirin Dose and ARU or Thrombosis}

The median initial aspirin dose was $40.5 \mathrm{mg} / \mathrm{d}$ (IQR 40.5-81), and the median normalized initial dose (initial dose/body weight, $\mathrm{mg} / \mathrm{kg} / \mathrm{d}$ ) was $6.3 \mathrm{mg} / \mathrm{kg} / \mathrm{d}$ (IQR 4.0-8.7). Median initial ARU by VerifyNow testing was 468 ARU (IQR 427-520). By multivariable logistic regression analysis, we were unable to detect significant correlation between initial normalized dose and therapeutic platelet inhibition on initial testing $(P=.10)$ (Table 2). The rate of thrombosis was not significantly different between single-ventricle and two-ventricle patients $(P=.77)$.

Thrombosis was detected in 2 of $366(0.6 \%)$ patients with therapeutic platelet inhibition (ARU < 550), and in 9 of 64 $(14 \%)$ patients who had platelet unresponsiveness (ARU $\geq 550$ ) on initial testing. Patients with and without thrombosis were not significantly different regarding sex $(P=.37)$, diagnosis $(P=.77)$, age $(P=.38)$, body weight $(P=.36)$, or initial aspirin dose $(P=.63)$ (Table 2). Initial ARU reading had an excellent correlation with thrombosis (AUC, 0.844; 95\% CI, 0.701-0.987, $P<.001$ ) and the optimal cut-off value of ARU $>553$ had an associated sensitivity of $82 \%$ (9 of 11 patients with thrombosis correctly classified) and an associated specificity of 88\% (369 of 419 control patients correctly classified) (Figure 2). Patients with thrombosis had significantly greater initial ARU readings ( $\geq 550$ ARU) compared with those without thrombosis (medians: 571 vs 465 ARU respectively, $P<.001$ ) (Figure 3, $A)$. Multivariable logistic regression confirmed that initial
TABLE 1. Patient characteristics $(n=430)$

\begin{tabular}{|c|c|}
\hline Characteristic & $\mathbf{n}$ \\
\hline Male, $\mathrm{n}(\%)$ & $252(59)$ \\
\hline Median age at surgery, y (IQR) & $1(0.25-3)$ \\
\hline Median weight, kg (IQR) & $9.1(4.6-14.9)$ \\
\hline Single ventricle, $\mathrm{n}(\%)$ & $212(49)$ \\
\hline \multicolumn{2}{|l|}{$\begin{array}{l}\text { Surgical procedure performed, } \\
\text { number of patients }\end{array}$} \\
\hline $\begin{array}{l}\text { Palliative surgery for single } \\
\text { ventricle }\end{array}$ & 148 \\
\hline $\begin{array}{l}\text { Coronary artery reconstruction/ } \\
\text { reimplantation }\end{array}$ & 52 \\
\hline $\begin{array}{l}\text { Complex valve repair/ } \\
\text { replacement }\end{array}$ & 157 \\
\hline $\begin{array}{l}\text { Pulmonary vein/SVC } \\
\text { reconstruction }\end{array}$ & 13 \\
\hline $\begin{array}{l}\text { Complex intracardiac baffle } \\
\text { (atrial or ventricular) }\end{array}$ & 21 \\
\hline $\begin{array}{l}\text { Complex aortic or pulmonary } \\
\text { artery reconstruction }\end{array}$ & 32 \\
\hline Ventricular assist device & 7 \\
\hline $\begin{array}{l}\text { Median initial aspirin dose, } \mathrm{mg} / \\
\mathrm{kg} / \mathrm{d}\end{array}$ & $6.3(4.0-8.7)$ \\
\hline Rate of ARU > 550, \% & $14.9(\mathrm{n}=64)$ \\
\hline Thrombosis rate (within $30 \mathrm{~d}$ ), $\%$ & $2.6(n=11)$ \\
\hline \multicolumn{2}{|l|}{$\begin{array}{l}\text { Location of thrombosis, number } \\
\text { of patients }\end{array}$} \\
\hline Femoral artery & 2 \\
\hline $\begin{array}{l}\text { Fontan pathway, Baffle, and } \\
\text { fenestration }\end{array}$ & 1 \\
\hline Shunt thrombosis & 2 \\
\hline Embolic stroke & 2 \\
\hline Left ventricle & 1 \\
\hline Right atrium/SVC/IJ & 2 \\
\hline Coronary artery anastomosis & 1 \\
\hline
\end{tabular}

ARU was the only independent risk factor for thrombosis after we adjusted for weight and initial aspirin dose as covariates with an odds ratio of 30.1 (95\% CI, 6.3-144.3, $P<.001$, Table 2). Multivariable logistic regression model testing showed reasonably good model calibration, as indicated by a nonsignificant Hosmer-Lemeshow test $\left(\chi^{2}=4.08\right.$ on 8 degrees of freedom, $\left.P=.849\right)$.

\section{Relationship Between Aspirin Dose Adjustment and Thrombosis Among Patients With ARU $\geq \mathbf{5 5 0}$}

Forty of $64(63 \%)$ patients with ARU $\geq 550$ were subjected to aspirin dose escalation. Aspirin dose escalation resulted in a significant decrease in ARU from $603 \pm 45$ to $469 \pm 68(P<.001)$ (Figure 3, B). In these 40 patients, the normalized dose was increased from median of $6.1 \mathrm{mg} / \mathrm{kg} / \mathrm{d}$ to $10 \mathrm{mg} / \mathrm{kg} / \mathrm{d}$. Twenty-four of $64(33 \%)$ 
TABLE 2. Clinical characteristics, aspirin dose, and ARUs in patients with and without thrombosis

\begin{tabular}{|c|c|c|c|c|}
\hline Characteristic & $\begin{array}{c}\text { Thrombosis } \\
(\mathbf{n}=11)\end{array}$ & $\begin{array}{c}\text { No thrombosis } \\
(n=419)\end{array}$ & $\begin{array}{c}\text { Univariate } \\
P \text { value } \\
\end{array}$ & $\begin{array}{c}\text { Multivariable } \\
\text { OR }(95 \% \text { CI }), P \text { value }\end{array}$ \\
\hline Sex & & & .37 & \\
\hline Male & $5(46 \%)$ & $247(59 \%)$ & & \\
\hline Female & $6(54 \%)$ & $171(41 \%)$ & & \\
\hline Diagnosis & & & .77 & \\
\hline Single ventricle & $5(46 \%)$ & $211(50 \%)$ & & \\
\hline Biventricular & $6(54 \%)$ & $208(50 \%)$ & & \\
\hline Age, mo & $7(1-36)$ & $12(3-36)$ & .38 & \\
\hline Weight, kg & $7.2(3.3-12.3)$ & $9.3(4.6-15.0)$ & .36 & $P=.73$ \\
\hline Initial aspirin dose, $\mathrm{mg} / \mathrm{kg} / \mathrm{d}$ & $5.7(2.6-7.8)$ & $6.3(4.1-8.8)$ & .63 & $P=.69$ \\
\hline Initial reading, ARU & $571(555-626)$ & $465(425-517)$ & $<.001$ & $\begin{array}{c}30.1(6.3-144) \\
P<.001^{*}\end{array}$ \\
\hline
\end{tabular}

Continuous data are given as median (interquartile range). $O R$, Odds ratio; $C I$, confidence interval; $A R U$, aspirin reaction units. ${ }^{*}$ Significant association between initial ARU reading and thrombosis independent of weight and indexed initial aspirin dose.

patients with ARU $\geq 550$ did not undergo testing-based dose escalation as the result of provider preference.

Among the subset of 64 patients with initial ARU $\geq 550$, we examined the association between aspirin dose increase and subsequent thrombosis. In this subgroup analysis, patients with thrombosis $(\mathrm{n}=9)$ and without thrombosis $(\mathrm{n}=55)$ were not significantly different regarding age $(P=.40)$, weight $(P=.48)$, sex $(P=.47)$, diagnosis $(P=1.00)$, or initial aspirin dose (adjusted to body weight) $(P=.99)$ (Table 3). None of the 40 patients who had an increase in their initial aspirin dose had thrombosis $(0 \%$; $95 \%$ CI, $0 \%-10 \%$ ), whereas 9 of the 24 patients who did not have an aspirin dose increase developed thrombosis

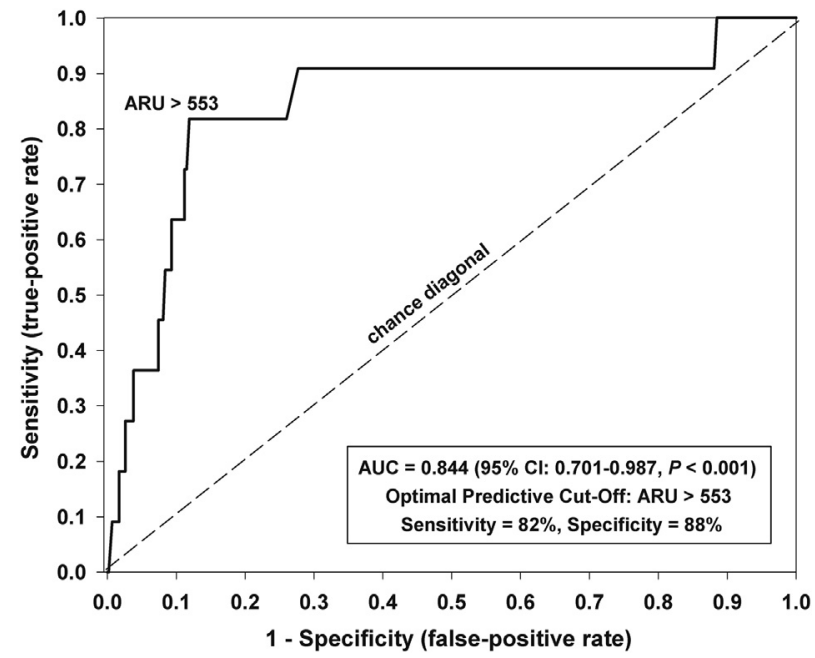

FIGURE 2. Empirical ROC curve showing the relationship between initial ARU reading and thrombosis with AUC of $0.844(P<.001)$. The cut-off value of ARU $>553$ is based on the point on the curve farthest from the chance diagonal (Youden J-index) for balancing sensitivity and specificity of association between ARU and thrombosis. The cut-off value is associated with a sensitivity of 0.82 and a specificity of 0.88 . ARU, Aspirin reaction units; $A U C$, area under the curve; $C I$, confidence interval.
(38\%; 95\% CI, 20\%-57\%). Thus, none of the 9 patients with thrombosis had a dose increase, whereas 40 of the 55 patients without thrombosis $(73 \%)$ had an increased dose of aspirin and remained free from thrombosis $(P<.001$, Table 3). When we adjusted for weight in multivariable logistic regression (Table 3), the only significant independent risk factor for thrombosis was lack of increase in aspirin dose $(P<.001)$. Because there were no thrombosis events among patients who had dose adjustment, an estimated odds ratio was calculated by assuming one thrombosis event in that cohort, yielding an adjusted odds ratio of 23.6 (95\% CI, 2.7-202.8, $P<.001)$.

\section{Correlation Between VerifyNow and TEG-PM}

In a subset cohort $(\mathrm{n}=31)$, platelet inhibition by the VerifyNow system was compared with values obtained by TEG-PM. The Pearson correlation indicated a strong negative correlation between ARU and percent inhibition by TEG-PM $(\mathrm{r}=-0.88, P<.001)$, demonstrating that patients with lower ARU values had a greater percent inhibition by TEG-PM. The proportion of patients who had a response of greater than $50 \%$ AA inhibition by TEG-PM was $100 \%$ (22/22) among patients with ARU $<550$ compared with only $14 \%$ (1/7) for those with ARU values $\geq 550(P<.001$, Fisher exact test, Figure 4$)$.

\section{Incidence of Aspirin Resistance}

A total of 45 patients with initial ARU $\geq 550$ ultimately underwent dose escalation (including 5 of 9 patients who subsequently underwent dose increase after thrombosis event). Repeat platelet testing was performed after dose escalation in 23 of 45 patients $(51 \%)$ with initial ARU $\geq 550 ; 2$ patients demonstrated continued ARU $\geq 550$ despite maximal dose escalation. Thus, the estimated rate of aspirin resistance (persistent ARU $\geq 550$ despite maximal dose escalation) observed in this study 
Scatter Plot of Initial ARU Reading in Patients With and Without Thrombosis

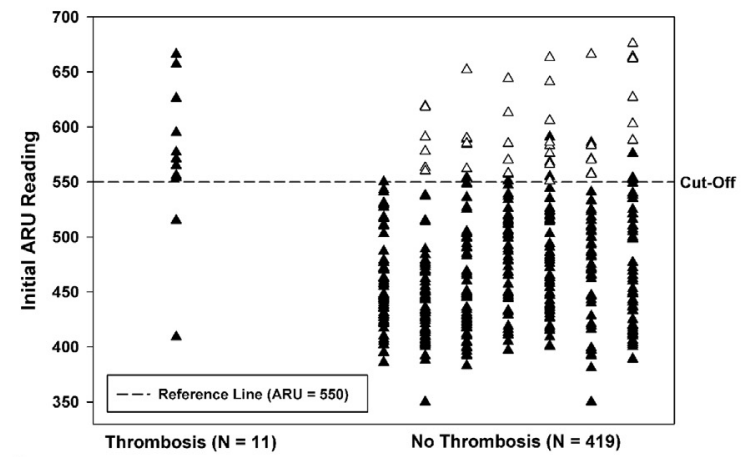

A

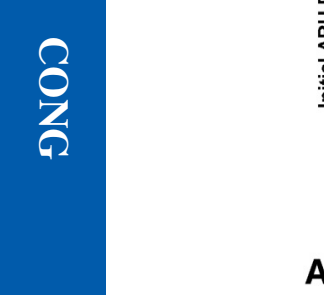

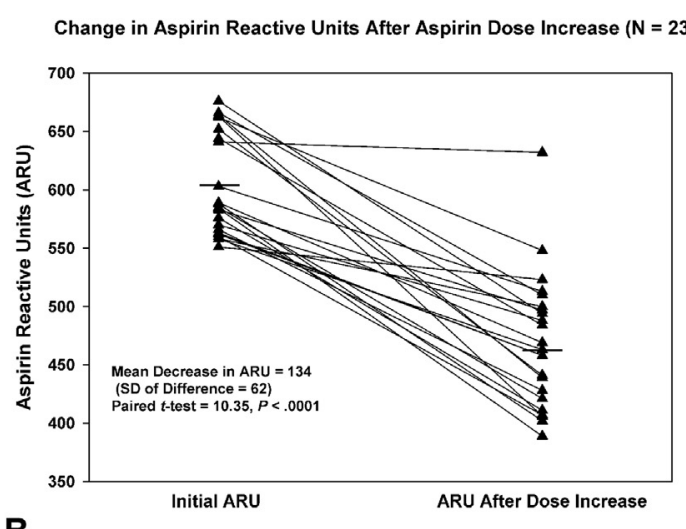

B

FIGURE 3. A, Scatterplot of initial ARU reading amongst patients with and without thrombosis: Open triangles represent patients with initial ARU $\geq 550$ who underwent dose escalation. Closed triangles represent patients who did not undergo dose adjustment following platelet testing. B, Change in ARU after aspirin dose increase demonstrating significant decrease in ARU after aspirin dose escalation in patients with initial ARU $\geq 550$. Mean decrease in ARU was $134 \pm 62(P<.001)$. ARU, Aspirin reaction units.

was 2 of $389(0.5 \%)$ patients, excluding the 41 patients with ARU $\geq 550$ who neither had repeat testing nor dose escalation.

\section{Thrombosis Before Initiation of Aspirin}

Twenty-three patients who experienced thrombosis before the initiation of aspirin were excluded from the primary analysis. Median time between surgery and aspirin administration in the 23 patients was 7 days (IQR, 2-15). Duration between surgery and aspirin administration was significantly longer in 23 patients who experienced thrombosis prior to aspirin compared with 430 patients who did not (median 3 days; IQR, 2-5, $P<.05$ ).

\section{DISCUSSION}

Aspirin commonly is used for thrombosis prophylaxis after specific cardiac surgical procedures, and this study highlights the variability in platelet inhibition after administration of aspirin at standard dose of 3 to $10 \mathrm{mg} / \mathrm{kg} /$ d, with $15 \%$ of patients demonstrating ARU $\geq 550$ by VerifyNow testing. Although no correlation was detected between the indexed aspirin dose and outcomes of ARU or thrombosis, a significant correlation between initial ARU $\geq 550$ and thrombosis was detected. Patients undergoing dose escalation based on aspirin testing demonstrated lower rate of thrombosis compared with patients who did not undergo testing-based dose adjustment. Dose increase resulted in subsequent $\mathrm{ARU}<550$ on repeat testing in most patients, with 2 patients demonstrating confirmed aspirin resistance (persistent $\mathrm{ARU} \geq 550$ despite maximum aspirin dose of $10 \mathrm{mg} / \mathrm{kg} / \mathrm{d}$ ).

This study demonstrates significant variability in platelet response to standard weight-based dosing of aspirin and supports the findings of previous studies. ${ }^{8,15}$ The mechanisms underlying this variability were not ascertained in this study, although they require future

TABLE 3. Subgroup analysis of patients with initial ARU $\geq 550$ : effect of dose increase

\begin{tabular}{|c|c|c|c|c|}
\hline Characteristic & $\begin{array}{c}\text { Thrombosis } \\
\quad(\mathbf{n}=\mathbf{9})\end{array}$ & $\begin{array}{l}\text { No thrombosis } \\
\quad(\mathbf{n}=\mathbf{5 5})\end{array}$ & $\begin{array}{c}\text { Univariate } \\
P \text { value }\end{array}$ & $\begin{array}{c}\text { Multivariable } \\
\text { OR }(95 \% \mathrm{CI}), P \text { value }\end{array}$ \\
\hline Sex & & & .47 & \\
\hline Male & $3(33 \%)$ & $29(53 \%)$ & & \\
\hline Female & $6(67 \%)$ & $26(47 \%)$ & & \\
\hline Diagnosis & & & 1.00 & \\
\hline Single ventricle & $5(56 \%)$ & $29(53 \%)$ & & \\
\hline Biventricular & $4(44 \%)$ & $26(47 \%)$ & & \\
\hline Age, mo & $7(1-72)$ & $24(4-60)$ & .40 & \\
\hline Weight, kg & $7.2(3.1-25.8)$ & $12.0(5.4-17.6)$ & .48 & $P=.89$ \\
\hline Initial aspirin dose, $\mathrm{mg} / \mathrm{kg} / \mathrm{d}$ & $5.7(2.3-10.0)$ & $5.9(3.2-7.4)$ & .99 & \\
\hline Aspirin dose increase & & & $<.001$ & $23.6(2.7-203)$, \\
\hline Yes & $0(0 \%)$ & $40(73 \%)$ & & $P<.001^{*}$ \\
\hline No & $9(100 \%)$ & $15(27 \%)$ & & \\
\hline
\end{tabular}

Continuous data are given as median (interquartile range). $O R$, Odds ratio; $C I$, confidence interval. *Significant association between the action of increasing aspirin dose in patients with initial ARU $>550$ and lack of thrombosis. Estimated OR of 23.6 calculated by assuming 1 thrombosis event in the dose increase group. 




FIGURE 4. Scatterplot depicting correlation between platelet testing by VerifyNow and TEG devices. When methods for determining platelet function are compared, Pearson correlation indicates a strong negative correlation between ARU by VerifyNow and percent inhibition by TEG-platelet mapping $(P<.001)$. Black circles represent ARU $<550$, whereas red circles represent ARU $>550$. TEG, Thromboelastography; $A A$, arachidonic acid.

elucidation. A number of putative mechanisms have been identified, including poor enteral absorption, drug interactions (proton pump inhibitors), esterase mediated aspirin metabolism, polymorphisms of COX-1 or glycoprotein IIIa, platelet heterogeneity before aspirin, and high platelet turnover. ${ }^{16,17}$ Nonsteady-state platelet production or destruction may have significant impact on antiplatelet therapy. Multiple mechanisms may simultaneously contribute to aspirin unresponsiveness after cardiac surgery, related to profound inflammatory response and thrombocytopenia. ${ }^{18}$ The data highlight the importance of measuring platelet response by laboratory testing if antiplatelet effect is desired.

A significant association between ARU $\geq 550$ on initial testing and clinical outcome of postoperative thrombosis was demonstrated in this study. Further supporting the relationship between ARU and thrombosis risk was the finding that all of the thrombosis events occurred in patients with persistent $A R U \geq 550$, ie, those who did not undergo dose escalation based on aspirin testing. Among patients with initial $A R U \geq 550$ who were not subjected to dose adjustment, $38 \%$ eventually demonstrated thrombosis events. In contrast, patients with ARU $\geq 550$ who underwent dose increase based on aspirin testing did not develop subsequent thrombosis. These data suggest that measuring platelet function to attain a therapeutic response and customize antiplatelet therapy is potentially important in this population. However, this study was limited by its retrospective study design, and confirmation of these findings within a prospective randomized trial is warranted.

Although there is lack of consensus regarding the definition of aspirin resistance, this study demonstrates that in our cohort, initial ARU $\geq 550$ frequently can be overcome by dose escalation. Aspirin resistance, defined in this study as persistent ARU $\geq 550$ despite maximal dose, was uncommon. These data are consistent with a previously reported low incidence of laboratory-based aspirin resistance in children. ${ }^{19}$ This study was unable to determine the true incidence of aspirin resistance since significant number of patients with ARU $\geq 550$ did not undergo dose escalation or postescalation retesting. Other investigators have alternatively defined aspirin resistance as the development of thrombosis despite aspirin therapy but have not correlated thrombosis with laboratory testing. 6 The data in this study suggest that, in pediatric patients after cardiac surgery, aspirin resistance may be attributed to variability in platelet response to weight-based dosing and frequently may be overcome by testing-based dosing.

Aspirin unresponsiveness by VerifyNow testing was defined as value of greater than or equal to 550 ARU. This value was adopted from manufacturer recommendation, which was derived from adult experience. A previous study showed that the 550 threshold value correlated well with thrombosis events, and therefore this value was used for discrimination of unresponsiveness in this study. ${ }^{8}$ This study confirmed that a value of 553 ARU was appropriate for distinguishing responsiveness versus unresponsiveness in pediatric patients undergoing cardiac surgery (See Methods section). Correlation of ARU $\geq 550$ by VerifyNow with $>50 \%$ platelet inhibition by TEG-PM supports the use of ARU $\geq 550$ by VerifyNow or $>50 \%$ inhibition by TEG-PM as reasonable cut-off values in this cohort of patients.

Previous studies have shown a lack of benefit of aspirin responsiveness testing in adult populations with coronary artery disease. ${ }^{20,21}$ However, these studies included a combination of both low- and high-risk patients in contrast to this study, which only included patients who were considered to be high risk for thrombosis based on known risk factors. Many pediatric patients receive aspirin therapy after cardiac surgery, even if risk of thrombosis is low. In these patients, platelet inhibition testing may not be warranted, as the benefit of aspirin responsiveness testing in low-risk populations has not been demonstrated.

A significant number of patients experienced thrombosis before the initiation of aspirin, and the duration between surgery and initiation of aspirin was prolonged in this subset of patients compared with those who did not experience pre-aspirin thrombosis. This suggests that the inability to initiate aspirin therapy early in the postoperative period is associated with risk of thrombosis. Previous studies have supported the hypothesis that early initiation of aspirin following surgery may be associated with lower thrombosis rates. ${ }^{22}$ Strategies to prevent these thrombosis events might include earlier initiation of aspirin, more aggressive anticoagulation, or introduction of intravenous antiplatelet agents as a bridge to aspirin therapy. ${ }^{23}$ 
On the basis of this study, the clinical protocol outlined in Figure 1 has been adopted widely at our institution to guide management of antiplatelet therapy in pediatric patients after cardiac surgery. If a patient is deemed to be high risk for thrombosis (based on procedural inclusion criteria) and does not exhibit contraindications to aspirin administration, aspirin is initiated at 3 to $10 \mathrm{mg} / \mathrm{kg} /$ $\mathrm{d}$ (only exception is that neonates receive a minimum of $40.5 \mathrm{mg}$ ) orally on cessation of bleeding and return of bowel function. VerifyNow testing is performed after at least 2 doses of aspirin and adjusted according to the algorithm.

The results of VerifyNow correlated well with results of TEG-PM, and thus either method could be used for guiding aspirin dose adjustment. This study used VerifyNow for several reasons. VerifyNow requires approximately $2 \mathrm{~mL}$ of blood, whereas TEG-PM requires approximately 4 to $6 \mathrm{~mL}$ of blood with the current technology. Particularly in small children and neonates, blood volume required for testing is of significant concern. Second, TEG requires a skilled technician and CLIA-certified laboratory, whereas VerifyNow is an automated test (CLIA waived) and can be performed point-of-care (inpatient unit or outpatient office).

There are several limitations to this study. The incidence of thrombosis reported in the study likely underestimates the true incidence of thrombosis in postsurgical patients because patients experiencing thrombosis before the initiation of aspirin as well as those requiring multimodal therapy were excluded from the study. In addition, thrombosis within 30 days after surgery was used as an outcome measure, and thrombosis was included only if detected in large vessels by echocardiogram; systematic surveillance for peripheral vascular thrombosis was not performed. The power of this study was limited by small number of overall thrombosis events as well as the small number of initially unresponsive patients who did not undergo a dose increase. Because clinicians were not blinded to the results of platelet inhibition testing, it is possible that patients with inadequate platelet inhibition who did not undergo dose escalation were subjected to closer surveillance, thus leading to a greater rate of detection of thrombosis.

In conclusion, this study demonstrates that platelet unresponsiveness to aspirin is associated with risk of thrombosis after high-risk pediatric cardiac surgery. Aspirin dosing based on weight yields inadequate platelet inhibition in approximately $15 \%$ of patients. In patients who demonstrate initial platelet unresponsiveness, immediate aspirin dose escalation is associated with reduced risk of thrombosis. Prospective randomized trials are necessary to demonstrate efficacy of aspirin responsiveness testing in reducing rate of postoperative thrombosis in this population.

\section{Conflict of Interest Statement}

Authors have nothing to disclose with regard to commercial support.

\section{References}

1. Manlhiot C, Menjak IB, Brandao LR, Gruenwald CE, Schwartz SM, Sivarajan VB, et al. Risk, clinical features, and outcomes of thrombosis associated with pediatric cardiac surgery. Circulation. 2011;124:1511-9.

2. Li JS, Yow E, Berezny KY, Rhodes JF, Bokesch PM, Charpie JR, et al. Clinical outcomes of palliative surgery including a systemic-to-pulmonary artery shunt in infants with cyanotic congenital heart disease: does aspirin make a difference? Circulation. 2007;116:293-7.

3. Michelson AD. Methods for the measurement of platelet function. Am J Cardiol. 2009; 103:20A-6A.

4. Cholette JM, Mamikonian L, Alfieris GM, Blumberg N, Lerner NB Aspirin resistance following pediatric cardiac surgery. Thromb Res. 2010;126:200-6.

5. Heistein LC, Scott WA, Zellers TM, Fixler DE, Ramaciotti C, Journeycake JM, et al. Aspirin resistance in children with heart disease at risk for thromboembolism: prevalence and possible mechanisms. Pediatr Cardiol. 2008;29:285-91.

6. Freedman JE. The aspirin resistance controversy: clinical entity or platelet heterogeneity? Circulation. 2006;113:2865-7.

7. Frelinger AL III, Furman MI, Linden MD, Li Y, Fox ML, Barnard MR, et al. Residual arachidonic acid-induced platelet activation via an adenosine diphosphate-dependent but cyclooxygenase-1- and cyclooxygenase-2independent pathway: a 700-patient study of aspirin resistance. Circulation. 2006;113:2888-96.

8. Emani S, Trainor B, Zurakowski D, Baird CW, Fynn-Thompson FE, Pigula FA, et al. Aspirin unresponsiveness predicts thrombosis in high-risk pediatric patients after cardiac surgery. J Thorac Cardiovasc Surg. 2014;148:810-4.

9. Collet JP, Cuisset T, Range G, Cayla G, Elhadad S, Pouillot C, et al. Bedside monitoring to adjust antiplatelet therapy for coronary stenting. $N$ Engl J Med. 2012;367:2100-9.

10. Newcombe RG. Two-sided confidence intervals for the single proportion: comparison of seven methods. Stat Med. 1998;17:857-72.

11. Katz MH. Multivariable Analysis: A Practical Guide for Clinicians and Public Health Researchers. 3rd ed. Cambridge; New York: Cambridge University Press; 2011:140-61.

12. Zhou X-h, McClish DK, Obuchowski NA. Statistical Methods in Diagnostic Medicine. 2nd ed. Hoboken, NJ: Wiley; 2011:24-55.

13. Harrell FE Jr. Regression Modeling Strategies: With Applications to Linear Models, Logistic Regression, and Survival Analysis. New York: Springer; 2001:247-67.

14. McCullagh P, Nelder JA. Generalized Linear Models. 2nd ed. Boca Raton, Fla: Chapman \& Hall/CRC Press; 1998:101-24.

15. Mir A, Frank S, Journeycake J, Wolovitis J, Guleserian K, Heistein L, et al. Aspirin resistance in single-ventricle physiology: aspirin prophylaxis is not adequate to inhibit platelets in the immediate postoperative period. Ann Thorac Surg. 2015;99:2158-64.

16. Floyd CN, Ferro A. Mechanisms of aspirin resistance. Pharmacol Ther. 2014; 141:69-78.

17. Frelinger AL III, Li Y, Linden MD, Barnard MR, Fox ML, Christie DJ, et al. Association of cyclooxygenase-1-dependent and -independent platelet function assays with adverse clinical outcomes in aspirin-treated patients presenting for cardiac catheterization. Circulation. 2009;120:2586-96.

18. Floyd CN, Ferro A. Antiplatelet drug resistance: molecular insights and clinical implications. Prostaglandins Other Lipid Mediat. 2015;120:21-7.

19. Yee DL, Dinu BR, Sun CW, Edwards RM, Justino H, Teruya J, et al. Low prevalence and assay discordance of "aspirin resistance" in children. Pediatr Blood Cancer. 2008;51:86-92.

20. Bolliger D, Filipovic M, Matt P, Tanaka KA, Gregor M, Zenklusen U, et al, Reduced aspirin responsiveness as assessed by impedance aggregometry is not associated with adverse outcome after cardiac surgery in a small low-risk cohort. Platelets. 2016;27:254-61.

21. Breet NJ, van Werkum JW, Bouman HJ, Kelder JC, Ruven HJ, Bal ET, et al. Comparison of platelet function tests in predicting clinical outcome in patients undergoing coronary stent implantation. JAMA. 2010;303:754-62.

22. Heidari-Bateni G, Norouzi S, Hall M, Brar A, Eghtesady P. Defining the best practice patterns for the neonatal systemic-to-pulmonary artery shunt procedure. J Thorac Cardiovasc Surg. 2014;147:869-73.e3.

23. Emani S, Kaza AK, Almodovar M, Thiagarajan R, Emani SM. Intravenous GPIIb/IIIa inhibitor for secondary prevention of shunt thrombosis in a pediatric patient. Ann Thorac Surg. 2015;99:e151-3.

Key Words: thrombosis, aspirin, platelet function, cardiac surgery, pediatrics 